\title{
A Case of Familial Mediterranean Fever Presenting with Pseudo-Bartter Syndrome
}

\author{
Can Hüzmeli ${ }^{1, *}$, Emine Uslu Yurteri ${ }^{2}$, Mustafa Bardakçı ${ }^{3}$ \\ ${ }^{1}$ Department of Nephrology, Hatay Education and Research Hospital, Hatay, Turkey. \\ ${ }^{2}$ Department of Rheumatology, Hatay Education and Research Hospital, Hatay, Turkey. \\ ${ }^{3}$ Department of Radiology, Hatay Education and Research Hospital, Hatay, Turkey.
}

How to cite this paper: Can Hüzmeli, Emine Uslu Yurteri, Mustafa Bardakçı. (2021) A Case of Familial Mediterranean Fever Presenting with Pseudo-Bartter Syndrome. International Journal of Clinical and Experimental Medicine Research, 5(3), 353-355. DOI: 10.26855/ijcemr.2021.07.019

Received: May 24, 2021

Accepted: June 18, 2021

Published: July 1, 2021

*Corresponding author: Can Hüzmeli, Department of Nephrology, Hatay Education and Research Hospital, Hatay, Turkey.

Email: chuzmeli@hotmail.com

\begin{abstract}
Bartter Syndrome is a disease (sporadic or familial) characterized by hypochloremia, hypokalemia, hyponatremia, metabolic alkalosis, hyperaldosteronism, hyperreninemia and normal blood pressure. Pseudo-Bartter syndrome is characterized by hypochloremia associated with metabolic alkalosis and hypokalemia without any pathology in the tubular transport mechanism. Bartter syndrome and pseudo bartter syndrome have similar biochemical profile. Pseudo-Bartter syndrome is different from Bartter syndrome as extrarenal electrolyte loss occurs. Pseudo-Bartter syndrome may develop as a result of cystic fibrosis, inappropriate diuretic use, chlorine-poor diet, bulimia, cyclic vomiting, and laxative abuse. In the treatment of pseudo-bartter syndrome, the treatment of the underlying disease and the provision of fluid and electrolyte balance. Familial Mediterranean fever is characterized by non-infectious recurrent episodes of fever and serositis and is an autosomal recessive genetic disease that can develop amyloidosis over time. In this paper, we made the diagnosis of Familial Mediterranean fever in an 18-year-old male patient who presented with pseudo-Bartter syndrome. The association of pseudo-Bartter syndrome and Familial Mediterranean fever has not been previously described in the literature.
\end{abstract}

\section{Keywords}

Pseudo-Bartter Syndrome, Hypokalemia, Familial Mediterranean Fever

\section{Introduction}

Bartter syndrome is characterized with hypokalemic metabolic alkalosis, hyperreninemia, increased aldosterone secretion, and normal blood pressure. In Bartter syndrome, growth retardation, vomiting, polyuria, polydipsia, hypochloremia, metabolic alkalosis, hypokalemia, hypomagnesemia, and hypercalciuria can be seen. Bartter syndrome is autosomal recessive. Bartter syndrome reduces the extracellular fluid volume with salt absorption disorder, and blood pressure is low or normal [1].

In Pseudo-Bartter Syndrome, metabolic alkalosis develops without pathology in tubular transport mechanisms. Pseudo-Bartter syndrome is also a group of diseases in which hypokalemic metabolic alkalosis is observed without primary renal disease. Causes of pseudo-bartter syndrome include cystic fibrosis, inappropriate diuretic use, chlorine-poor diet, bulimia, cyclic vomiting, laxative abuse, primary hyperaldosteronism, Cushing's syndrome, hypertrophic pyloric stenosis, and some medications (such as aminoglycoside, amphoteresin B) and nephrotoxic agents (such as heavy metal intoxication) counts [2-3]. 


\section{Case}

An 18-year-old male patient was admitted to the emergency room with complaints of weakness, fatigue, agitation and aggression. There was no feature in his personal background and family history. In the physical examination of the patient, blood pressure was $120 / 80 \mathrm{mmHg}$, pulse $80 / \mathrm{min}$, body temperature $36.5^{\circ} \mathrm{C}$, agitation and aggression, and other physical examination was normal. Glucose was $85 \mathrm{mg} / \mathrm{dl}$ (70-110), blood urea nitrogen $18 \mathrm{mg} / \mathrm{dl}$ (7-25), serum creatine $1.03 \mathrm{mg} / \mathrm{dl}$ (0.6-1.3), alanine amino transferase $218 \mathrm{IU} / \mathrm{L}$ (5 -42), aspartate amino transferase 127 IU/L (5.34), potassium 3.0mmol/L (3.5-5.5) sodium 134mmol/L (135-145), chlorine 77mmol/L (98-106), magnesium $2.5 \mathrm{mg} / \mathrm{dl}$ (1.58-2.5), leukocyte value 8.4 (4.5-11), hemoglobin 14.4 g/dl (13.5-17.5) platelet count 231,000 $\mathrm{mm}^{3}$ (150 -403000), CRP 0.46 (0-5), fibrinogen 295mg/dl (200-400) and serologically anti HAV negative, antiHBs positive, anti HCV was negative. Blood gas was evaluated as $\mathrm{Ph} 7.5, \mathrm{PCO}_{2} 57, \mathrm{HCO}_{3} 45 \mathrm{mmol} / \mathrm{L}$ metabolic alkalosis. Complete urinalysis revealed pH 8 and spot urine analysis revealed sodium $216 \mathrm{mEq} / \mathrm{L}$, potassium $11 \mathrm{mEq} / \mathrm{L}$, and chlorine $36 \mathrm{mEq} / \mathrm{L}$. The abdomen was reported as normal on ultrasonography. The patient was evaluated by neurology in the emergency, and brain tomography and brain magnetic resonance imaging were reported to be normal. In addition, the air density was evaluated as pneumomediastinum in the thorax tomography performed in the outer center, in the paratrachial area, in the mediastinum and in both hemithoraces.

When the patient was questioned after his agitation had passed, he had had abdominal pain for two years and had no other additional complaints. According to the patient, abdominal pain started to occur every 2 months and then once a month and lasted for two days. Recently, he has had agitation and aggression along with abdominal pain, harming himself and those around him. When he was admitted to the hospital, a psychiatric consultation was requested with these complaints. Potassium replacement, colchicine tablet and saline were given in the treatment of the patient. After the treatment, his complaints disappeared after 3 days, and his tests returned to normal. Genetic testing was requested for cystic fibrosis since the patient whose complaints resolved had pseudo-Bartter and pneumomediastinum. In addition, genetic testing was requested for familial Mediterranean fever. The patient was discharged with colchicine tablet $3 \times 1$. Control thoracic tomography of the patient was reported as normal. At the control two months later, he did not have any complaints, and his tests were normal. Genetic testing for cystic fibrosis was normal. Gene mutation was requested for familial Mediterranean fever, no mutation was detected.

\section{Discussion}

Familial Mediterranean fever is a self-limiting, autosomal recessive inherited autoinflammatory disease characterized by recurrent fever, peritonitis, pleuritis and arthritis. It is the most common hereditary autoinflammatory disease. It is especially common among Jews, Arabs, Turks, and Armenians. Familial Mediterranean fever is defined as the MEFV gene located on the short arm of the 16th chromosome which is responsible for the disease. None of the identified mutations may be detected in all Familial Mediterranean fever patients. The diagnosis of the disease is made as clinical. Genetic testing helps diagnosis. The main purpose of treatment is to reduce the frequency and severity of attacks. It is also to prevent the development of amyloidosis, which causes end-stage renal disease. The most important drug used in treatment is colchicine [4]. Hydration and potassium replacement was applied to the patient who presented with Pseudo-Bartter syndrome, and 3x0.5mg colchicine tablet was initiated. The genetic mutations we made for our patient's cystic fibrosis, and Familial Mediterranean fever were detected as negative. In the follow-up of the patient 2 months later, the abdominal pain did not recur, and the laboratory tests were normal.

In the majority of cases, Bartter syndrome is usually associated with electrolyte abnormalities, including hypokalemia and metabolic alkalosis. In some patients, magnesium and phosphate levels decrease and serum renin and aldosterone levels increase. The urine shows high excretion of sodium, potassium and PGE2. It is associated with 24-hour high urine calcium excretion. Hypercalciuria supports the diagnosis of Bartter syndrome and helps exclude Gitelman syndrome. Spot urine chloride concentration is usually greater than 35 meq/L. In the pathogenesis of Bartter syndrome, a primary inherited defect in reabsorption in the thick arm of the henle loop via the $\mathrm{Na}+-\mathrm{K}+$ -2Cl- pump is caused by renal excretion of sodium, potassium, and chloride. Imperfect $\mathrm{NaCl}$ reabsorption in the outgoing arm of the Henle loop leads to increased delivery of $\mathrm{NaCl}$ to distal tubules and thus aldosterone-mediated reabsorption of $\mathrm{Na}+$ in exchange for $\mathrm{K}+$ and $\mathrm{H}+$. This results in hypokalemia and metabolic alkalosis. Increased delivery of $\mathrm{NaCl}$ to macula densa activates tubuloglomerular feedback and increases the production of prostaglandins. Paracellular reabsorption of $\mathrm{Ca}$ and $\mathrm{Mg}$ is also impaired and causes hypocalcemia and hypomagnesemia. Prostaglandin E2 excess and severe potassium depletion may also impair the reabsorption of $\mathrm{Na}+-\mathrm{K}+-2 \mathrm{Cl}-$ [5-6]. Our patient had low urinary potassium excretion, but urinary sodium and chlorine excretion was high.

The cause of metabolic alkalosis is often revealed at the initial evaluation of patients with a careful history and 
physical examination. In the diagnosis of metabolic alkalosis, in addition to blood gas analysis, plasma potassium and chloride levels are important in confirming the diagnosis. It is easy to diagnose metabolic alkalosis, the important thing is to be able to make the differential diagnosis of pathologies that form metabolic alkalosis. At this point, it is important to determine the urine chloride level to facilitate the differential diagnosis and to guide our treatment. If urine chlorine level is low, it is known as chlorine sensitive metabolic alkalosis. Urine is resistant to chlorine in cases with high chlorine (e.g. Bartter syndrome, Gitelman syndrome, diuretic use).

In conclusion, Bartter syndrome and pseudo-Bartter syndrome should be considered in patients with hypokalemia and metabolic alkalosis. It is recommended to diagnose and start treatment immediately in children and young adults. Although it is rarely seen in adults, common causes such as diuretics and vomiting should be considered after ruling out. Our case is the first familial Mediterranean fever case presenting with pseudo-Bartter syndrome.

\section{Funding}

This article has not received support from any institution.

\section{References}

[1] Heilberg, I. P., Tótoli, C., Calado, J. T. (2015). Adult presentation of Bartter syndrome type IV with erythrocytosis. Einstein (Sao Paulo), 2015, 13(4): 604-606. https://doi.org/10.1590/S1679-45082015RC3013.

[2] Gürsu, M., Öztürk, S., Aydin, Z., Uzun, S., Kazancioğlu, R. (2009). Ağır Metabolik Alkalozla Seyreden Bir Pseudo-Bartter Olgusu (A Case of Pseudo-Bartter Syndrome with Severe Metabolic Alkalosis). Turkish Nephrology, Dialysis and Transplantation Journal, 2009, 18: 136-139.

[3] da Silva Cunha, T., Heilberg, I. P. "Bartter syndrome: causes, diagnosis, and treatment.” International Journal of Nephrology and Renovascular Disease, 2018, 11: 291. 10.2147/IJNRD.S155397.

[4] Migita, K., Asano, T., Sato, S., Koga, T., Fujita, Y., Kawakami, A. (2018). "Familial Mediterranean fever: overview of pathogenesis, clinical features and management.” Immunological Medicine, 41.2(2018): 55-61.3. https://doi.org/10.1080/13497413. 2018.1481579.

[5] Kumar, J. S. (2020). “Bartter Syndrome.” Asian Journal of Nursing Education and Research, 2020, 10(4): 509-511.

[6] Mantoo, M. R., Kabra, M., Kabra, S. K. (2020). “Cystic Fibrosis Presenting as Pseudo-Bartter Syndrome: An Important Diagnosis that is Missed!” Indian J Pediatr., 2020. https://doi.org/10.1007/s12098-020-03342-8. 